\title{
Fitness costs and stability of Cry1Fa resistance in Brazilian populations of Spodoptera frugiperda
}

\author{
Oscar F Santos-Amaya, ${ }^{a}$ Clébson S Tavares,, João Victor C Rodrigues, ${ }^{a}$ \\ Silverio 0 Campos, ${ }^{a}$ Raul Narciso C Guedes, ${ }^{a}$ Analiza P Alves ${ }^{b}$ and \\ Eliseu José G Pereira ${ }^{a, c^{*}}$
}

\begin{abstract}
BACKGROUND: The presence of fitness costs of resistance to Bacillus thuringiensis (Bt) insecticidal proteins in insect populations may delay or even reverse the local selection of insect resistance to $B t$ transgenic crops, and deserves rigorous investigation. Here we assessed the fitness costs associated with Cry1Fa resistance in two strains of fall armyworm, Spodoptera frugiperda (Lepidoptera: Noctuidae), derived from field collections in different Brazilian regions and further selected in the laboratory for high levels of resistance to Cry1Fa using leaves of TC1507 corn.

RESULTS: Fitness components were compared using paired resistant and susceptible strains with similar genetic backgrounds and $F_{1}$ generations from reciprocal crosses, all of them reared on non-transgenic corn leaves. No apparent life history costs in the larval stage were observed in the Bt-resistant strains. Moreover, the resistance remained stable for seven generations in the absence of selection, with no decrease in the proportion of resistant individuals. Larval respiration rates were also similar between resistant and susceptible homozygotes, and heterozygotes displayed respiration rates and demographic performance equal or superior to those of susceptible homozygotes.
\end{abstract}

CONCLUSION: In combination, these results indicate the lack of strong fitness costs associated with resistance to Cry1Fa in the fall armyworm strains studied. These findings suggest that Cry1Fa resistance in S. frugiperda populations is unlikely to be counterselected in Cry1Fa-free environments.

(C) 2016 Society of Chemical Industry

Supporting information may be found in the online version of this article.

Keywords: Bt resistance; pleiotropic effects; fall armyworm; isogenic strains; life-history traits; intrinsic rate of population increase; resistance management

\section{INTRODUCTION}

Acquisition of adaptation to a new environment, such as insecticide resistance, may result in phenotypic changes deleterious to the organism in the absence of the xenobiotic. ${ }^{1-3}$ Costs associated with insecticide resistance are not always detected, and in some instances the fitness of resistant individuals is actually enhanced in the absence of the insecticide. ${ }^{1,3}$ For resistance to Bacillus thuringiensis $(B t)$ insecticidal proteins produced in transgenic plants, fitness costs may play an important role in the rate of resistance evolution in environments free of $B t$ proteins (i.e. refuge areas), ${ }^{4,5}$ although the occurrence and magnitude of trade-offs between $B t$ resistance and insect fitness vary widely. ${ }^{6}$

When developing pest-specific insect resistance management strategies, it is valuable to understand the potential for fitness costs to delay or potentially reverse evolution of local resistance in pest populations. ${ }^{4,7,8}$ When present, fitness costs reduce the coefficient of selection, favoring susceptible individuals relative to resistant individuals in refuge areas. ${ }^{8}$ Under these conditions, refuge and other environments without $B t$ proteins select against resistance, ${ }^{6}$ which helps to decrease the spread and frequency of resistance alleles in field populations. ${ }^{6,7,9}$ Another factor that modulates resistance evolution is the dominance level of fitness costs, which can be recessive, affecting only homozygous resistant individuals, or non-recessive (e.g. additive or dominant), affecting both homozygous resistant and heterozygous genotypes. , $^{4,8,10}$ As the majority of resistance alleles are carried by heterozygous individuals when resistance first evolves in a population, non-recessive

\footnotetext{
* Correspondence to: EJG Pereira, Departamento de Entomologia, Universidade Federal de Viçosa, Viçosa 36570-000, MG, Brazil.

E-mail: eliseu.pereira@ufv.br

a Departamento de Entomologia, Universidade Federal de Viçosa, Viçosa, MG, Brazil

b DuPont Pioneer, Johnston, IA, USA

c Instituto Nacional de Ciência e Tecnologia em Interações Planta-Praga, Universidade Federal de Viçosa, Viçosa, MG, Brazil
} 
fitness costs, those affecting heterozygotes, are most effective at delaying resistance. ${ }^{6}$ Therefore, accurate estimates of the fitness cost associated with resistance and its dominance level are important to understand the evolution of resistance and define resistance management measures to mitigate resistance to transgenic plants. , $^{4-10}$

Fitness costs are detected when one or more fitness components in resistant insects are impaired relative to susceptible ones or when the frequency of the resistance allele decreases over time in the absence of selection. ${ }^{6}$ In some studies, insect life history traits in resistant and susceptible strains are compared, ${ }^{11-15}$ and in other studies, the stability of resistance in the absence of exposure to $B t$ protein is also monitored..$^{13,14}$ Additionally, increase in the metabolic rate ${ }^{16}$ may indicate high energy demands to maintain the resistance mechanism; for example, production of enzymes that degrade the $B$ t protein ${ }^{17}$ or replacement of damaged epithelial cells in the insect gut to restore homeostasis, which may come at a cost for growth and reproduction.

The fall armyworm, Spodoptera frugiperda (Lepidoptera: Noctuidae), is a major polyphagous pest in corn as well as in other crops and has evolved resistance to transgenic Bt corn producing Cy1Fa in Puerto Rico, ${ }^{18}$ the United States ${ }^{19}$ and Brazil. ${ }^{20}$ These reported cases indicate a high risk for resistance evolution against $B t$ insecticidal proteins for managing $S$. frugiperda populations. Previous studies found that fitness costs of Cry1Fa resistance can be absent ${ }^{13-15}$ or present in fall armyworm strains, ${ }^{21}$ indicating the importance of conducting independent studies using populations of different genetic background.

Here we used two fall armyworm strains selected for high levels of resistance to Cry1Fa using corn leaves containing event TC1507. 22,23 Both strains originated from field collections in Brazilian states other than Bahia, ${ }^{22,23}$ where field-relevant resistance to this Bt corn and its fitness costs were characterized..$^{15,20}$ We show that the fall armyworm fitness of both Cry1Fa-resistant strains was not compromised on non-Bt corn leaves, and the implications of these findings for resistance management of $S$. frugiperda to Cry1Fa corn and other Bt crops are discussed.

\section{MATERIALS AND METHODS}

\subsection{Insect strains and rearing}

Two Cry1Fa-susceptible and two Cry1Fa-resistant strains of $S$. frugiperda were used in the experiments. The first susceptible strain (Lab-SS) was maintained in the laboratory in the absence of selection pressure with Bt proteins for over 15 years, while the second susceptible strain (MT) was derived from collections on non-Bt corn fields in four corn-producing Brazilian states in 2011. The first Cry1Fa-resistant strain (MTH) was derived from the MT strain by continuous exposure to TC1507. ${ }^{23}$ The second resistant strain (IrmaF) was derived from collections in TC1507 transgenic corn fields in two counties of Minas Gerais (Brazil), and selected for resistance to Cry1Fa using the TC1507 Bt corn event. $^{22}$ The resistance in these strains is incompletely recessive $\left(D_{x}=0.12-0.25\right)$, autosomal and without maternal effects. ${ }^{22,23}$ All of the strains were maintained at the Federal University of Viçosa (UFV), Brazil, and reared according to Kasten et al., ${ }^{24}$ with slight modifications.

\subsection{Sources of $B t$ and non-Bt corn leaf tissue}

Two corn hybrids commonly planted in Brazil were used: Bt corn $30 \mathrm{~F} 35 \mathrm{H}$ (event TC1507, producing Cry1 Fa) and its isogenic, non- $B t$ corn hybrid 30 F35 (Dupont Pioneer, Santa do Cruz do Sul, RS, Brazil). Corn hybrid seeds were sown in $4 \mathrm{~L}$ pots in the main experimental station of UFV using five seeds per pot and leaving four plants per pot after thinning. Plants were irrigated daily and fertilized at 10 and 35 days using $40 \mathrm{~g}$ of NPK 8-28-16 fertilizer per pot. Other cultivation practices were performed as recommended for growing corn in the region, ${ }^{25}$ without applying pesticides and with weeds managed manually. All experiments using plant materials were implemented using V4-V9-stage plants. Bt gene expression by the corn plants was checked using ImmunoStrip STX 10301/0050 test strips (Agdia Inc., Elkhart, IN) according to the manufacturer's instructions. All tests were as expected for the presence of the Cry1F protein (Bt plants) or its absence (non-Bt isoline plants).

\subsection{Preparing insects for fitness cost studies}

To minimize confounding effects related to genetic differences between susceptible and resistant populations, the Cry1Fa resistance trait from the IrmaF strain was introgressed into the Lab-SS strain using repeated rounds of recombination and selection. ${ }^{26,27}$ Briefly, the IrmaF strain was crossed with the susceptible strain, and the $F_{1}$ progeny was reared on non-Bt corn. Cry1Fa-resistant individuals were selected in the $F_{2}$ and $F_{3}$ generations on TC1507 $B t$ corn leaves. The $\mathrm{F}_{4}$ generation was backcrossed with the susceptible strain, and the progeny was reselected with Cry1Fa corn as described above. This backcross and selection process was repeated 3 times, as in Wang et al., ${ }^{28}$ producing an isogenic resistant strain named Lab-RR. As the Cry1Fa-resistant strain MTH was derived from the susceptible MT strain, ${ }^{23,29}$ no crosses were necessary. 27

Concentration-response bioassays using surface-treated larval diet $^{30}$ were conducted as described elsewhere ${ }^{22,23}$ to confirm the level of resistance to Cry1Fa attained by the new introgressed strain (Lab-RR) in relation to the parental susceptible and resistant strains. Briefly, seven concentrations of Cry1Fa plus a control treatment (i.e. no toxin) were applied to the surface of the rearing diet in 128-well trays (CD International, Pitman, NJ); one neonate ( $<24 \mathrm{~h}$ after hatching) was transferred to each well, and mortality and larval weight of survivors relative to controls (i.e. growth inhibition) were assessed after 7 days of toxin exposure. The Cry1 Fa protein used was supplied by Dr Marianne Carey (Case Western Reserve University, Cleveland, $\mathrm{OH}$ ). The protein was activated with trypsin, purified by high-performance liquid chromatography (HPLC), shipped in lyophilized form and stored at $-80^{\circ} \mathrm{C}$. The toxicity profile of the Cry1Fa stock used was similar to that reported elsewhere. ${ }^{18,23,31}$

In addition, survival rates from neonate to pupa were also compared among the three strains (IrmaF, Lab-RR and Lab-SS) using leaf tissue bioassays with Cry1Fa corn and its non-Bt isoline in the V4-V5 stage. A total of 160 individuals of each strain in 16 replications were assayed in a completely randomized experiment. Briefly, $340 \mathrm{mg}$ of leaf sections were placed in each well $(5.6 \times 3.6 \times 3 \mathrm{~cm})$ of 16 -well PVC trays (Advento do Brasil, Diadema, $\mathrm{SP})$, and ten neonates $(<24 \mathrm{~h}$ after hatching) were transferred to each well using a fine hair brush. After 7 days, the larvae were transferred singly to new trays (one larva per well), and leaf sections were replaced daily until pupation, when survival rates were recorded.

Using the four above-mentioned strains, the relative fitness of eight S. frugiperda phenotypes was compared: resistant (Lab-RR, MTH) and susceptible (Lab-SS, MT) to Cry1Fa, assumed to be homozygous resistant ( $r$ ) and susceptible (ss) respectively, 
and the progenies derived from reciprocal crosses between the resistant and susceptible strains (sor ${ }^{-}{ }^{-}$and sơro) respectively. To generate heterozygous armyworms, 120 pupae per strain (resistant and susceptible) were separated by sex. The adults were maintained in polyvinyl chloride (PVC) cages $(40 \mathrm{~cm}$ height $\times 30 \mathrm{~cm}$ diameter) for mating $\mathrm{S} Q \times \mathrm{R}^{\hat{\top}}$ and $\mathrm{S} \mathrm{o}^{+} \times \mathrm{R} Q$ and fed a solution of $10 \%$ sugar and $5 \%$ ascorbic acid soaked in cotton. ${ }^{24}$ The cages were lined with bond paper sheets to provide an oviposition substrate. The eggs were collected every other day for 4 days and placed in plastic bags $(35 \times 40 \mathrm{~cm})$ until hatching. The insects were maintained in a temperature-controlled environment set at $27 \pm 2{ }^{\circ} \mathrm{C}, 70 \pm 15 \%$ relative humidity and 14:10 h L:D photoperiod.

\subsection{Growth, development and pupal weight}

To assess existence of fitness costs associated with development, randomly selected neonates ( $<24 \mathrm{~h}$ after hatching) $(n=64)$ of

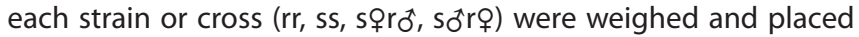
individually on non-Bt corn leaves arranged in 16-well PVC trays (Advento do Brasil, Diadema, São Paulo). Corn leaves were replaced every other day until insect pupation. Developmental time was calculated based on the day of hatching until pupation, and pupal weight and sex were assessed within $24 \mathrm{~h}$ of pupation. The growth rate $(\mathrm{GR})$ was calculated using the formula: $\mathrm{GR}=\left[W_{2}(\mathrm{mg})-W_{1}\right.$ $(\mathrm{mg})] / T$, where $W_{1}$ and $W_{2}$ are the weights of the neonates and pupae, respectively, and $T$ is the time in days from neonate to pupa. $^{21}$

\subsection{Reproductive rate}

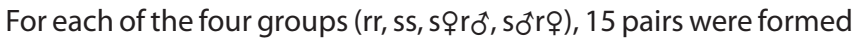
using virgin males and females obtained from the developmental time study. Each pair was placed in a PVC mating cage $(10 \mathrm{~cm}$ height $\times 10 \mathrm{~cm}$ diameter) and maintained as described above. The eggs produced by each female were collected daily until the end of the oviposition period (approximately 7 days) to estimate the fertility of each group. Egg masses were weighed and transferred to $200 \mathrm{~mL}$ plastic containers for hatching. Egg fertility was determined by counting the number of eggs hatched per egg mass daily.

\subsection{Life table-statistics}

Survival, development and reproduction data were used to estimate parameters describing the population growth potential of the four groups ( $r r, \mathrm{ss}, \mathrm{s}$ \&rô, sờ $\mathrm{r}$ ). Life table statistics, including the net reproductive rate (number of females produced per parental female, $R_{\mathrm{o}}$ ), mean generation time $(T)$, intrinsic rate of population increase (i.e. daily production of females per parental female, $r_{\mathrm{m}}$ ) and mean time for the population to double its size (doubling time, $D t$ ), were calculated ${ }^{32,33}$ using an SAS protocol previously described. ${ }^{34,35}$

\subsection{Larval respiration rate}

Respirometric assays were performed under laboratory conditions using a $\mathrm{TR} 3 \mathrm{C}$ respirometer equipped with a $\mathrm{CO}_{2}$ analyzer (Sable Systems International, Las Vegas, NV), as previously described, ${ }^{36}$ with slight modifications. To measure the mean respiratory rate ( $\mathrm{CO}_{2}$ production), 15 fourth instars ( 14 days old) from each group were randomly selected and individually placed in glass chambers of $25 \mathrm{~mL}$ volumetric capacity. The chambers were connected to a completely closed system for $3 \mathrm{~h}$ before scanning the $\mathrm{CO}_{2}$ produced by the insects $\left(\mu \mathrm{LCO}_{2}\right.$ insect $\left.{ }^{-1} \mathrm{~h}^{-1}\right)$, which was removed by
$\mathrm{CO}_{2}$-free airflow and measured using a $\mathrm{CO}_{2}$ infrared reader connected to the system.

\subsection{Resistance stability}

A resistance stability experiment was implemented to test whether the frequency of resistant individuals would decline over time using a selection-based stability analysis. ${ }^{13,14}$ Two lines (MT-RS and Lab-RS) originating from two sets of reciprocal crosses, MTH $\times$ MT and Lab-RR $\times$ Lab-SS, were used. $F_{1}$ adults of each set of reciprocal crosses were allowed randomly mate to in two PVC mating cages described previously to generate progenies with predicted homozygous susceptible (25\%) and resistant (25\%) as well as heterozygous genotypes (50\%) (defined as the $F_{1}$ generation of each line in the stability analysis). Both lines were maintained using a minimum population size of 200 adults during seven generations in the same conditions described for insect rearing.

To determine the frequency of resistant individuals, in each generation a portion of the neonates (256 of each line) were exposed to discriminating Cry1Fa concentrations that kill $99 \%$ of susceptible homozygous and heterozygous individuals using standard bioassays ${ }^{30}$ conducted using materials and the same conditions described above. The discriminating concentrations used for MT-RS and Lab-RS lines were 1978 and $2455 \mathrm{ng} \mathrm{cm}^{-2}$ Cry1Fa respectively. These concentrations kill 99\% ( $\left.\mathrm{LC}_{99}\right)$ of the susceptible homozygous and heterozygous individuals without affecting resistant homozygotes, which are not susceptible to Cry1Fa concentrations lower than $10000 \mathrm{ng} \mathrm{cm}^{-1} .^{23}$

\subsection{Statistical analysis}

Concentration-response bioassays carried out when preparing the insects for fitness comparisons were modeled with probit analysis ${ }^{37}$ using PoloPlus software, ${ }^{38}$ and $\mathrm{LC}_{50}$ and $\mathrm{EC}_{50}$ values obtained were used to estimate resistance ratios. Leaf tissue assays were analyzed using one-way analysis of variance to compare neonate-to-pupa survival among introgressed and parent strains on Cry1Fa corn and survival of the standard susceptible strain on non-Bt corn. Data from the diagnostic bioassays for stability of resistance were subjected to one-way analysis of variance to test whether survival rates in each line decreased during the seven generations without selection pressure. Homogeneity of variance and normality were checked for the above datasets (PROC MIXED, PROC UNIVARIATE, PROC GPLOT), ${ }^{35}$ and no transformation was needed.

Pupal weight, development time and relative growth rate were analyzed using a two-way analysis of variance with S. frugiperda group and sex as the main effects, and the means were separated using Fisher's least significant difference procedure $(P<0.05)$. Data on daily egg weight, number of eggs and neonates per female and respiratory rate $\left(\mu \mathrm{LCO}_{2}\right.$ insect $\left.{ }^{-1} \mathrm{~h}^{-1}\right)$ were subjected to a one-way analysis of variance, and the means were separated using Fisher's least significant difference $\left(P<0.05\right.$, PROC GLM). ${ }^{35}$ Residual analyses were performed for all response variables to assess whether the assumptions of homogeneity of variance and normality were met (PROC MIXED, PROC UNIVARIATE, PROC GPLOT), ${ }^{35}$ and no data transformation was needed.

The variances associated with the population growth parameters were estimated using the jackknife method ${ }^{39,40}$ using a SAS protocol developed by Maia et al. ${ }^{34}$ This procedure computes confidence intervals for all of the estimated life table parameters and $P$ values associated with unilateral or bilateral $t$-tests to perform pairwise comparisons between groups. 
Table 1. Toxicity of Cry1Fa to three Spodoptera frugiperda strains used to control for genetic background in the fitness cost study. IrmaF is a strain previously selected for resistance to the protein, ${ }^{22}$ Lab-SS is the laboratory standard susceptible strain and Lab-RR is the laboratory strain introgressed with the Cry1Fa resistance trait

\begin{tabular}{|c|c|c|c|c|c|}
\hline Response variable & Strain & Slope \pm SE & $\mathrm{LC}_{50}$ or $\mathrm{EC}_{50}(95 \% \mathrm{CL})^{\mathrm{a}}$ & Resistance ratio ${ }^{\mathrm{b}}$ & $\chi^{2}$ \\
\hline \multicolumn{6}{|l|}{ Mortality } \\
\hline & IrmaF & $n c^{c}$ & $>10000$ & $>183$ & $\mathrm{nc}^{\mathrm{c}}$ \\
\hline & Lab-RR & $n c^{c}$ & $>10000$ & $>183$ & $n c^{c}$ \\
\hline & Lab-SS & $2.08 \pm 0.25$ & $54.63(38.02-70.06)$ & 1 & $4.03^{*}$ \\
\hline \multicolumn{6}{|l|}{ Growth inhibition } \\
\hline & IrmaF & $n c^{c}$ & $>10000$ & $>2100$ & $\mathrm{nc}^{\mathrm{c}}$ \\
\hline & Lab-RR & $n c^{c}$ & $>10000$ & $>2100$ & $n c^{c}$ \\
\hline & Lab-SS & $1.74 \pm 1.08$ & $4.76(0.09-21.11)$ & 1 & $0.43^{*}$ \\
\hline \multicolumn{6}{|c|}{ 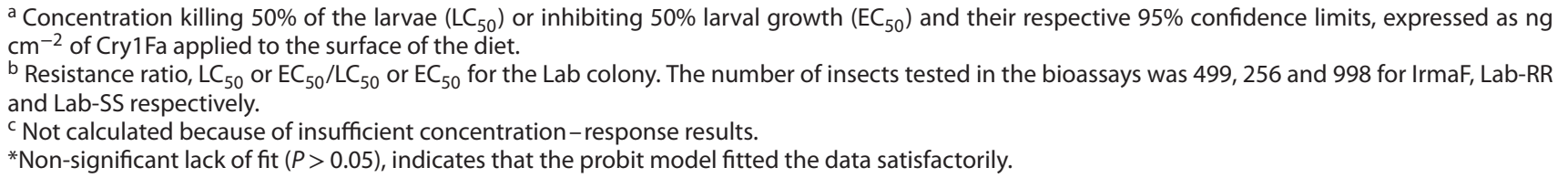 } \\
\hline
\end{tabular}

\section{RESULTS}

\subsection{Preparing insects for fitness cost studies}

An attempt was made to introgress the Cry1Fa resistance trait from the IrmaF strain into the susceptible laboratory population (Lab-SS) to obtain an isogenic strain named Lab-RR, which showed a level of resistance to Cry1Fa similar to that shown by the parent strain IrmaF in artificial diet (Table 1). Likewise, in leaf tissue bioassays, neonate-to-pupa survival on Cry1Fa corn for the resistant strains was not different from the survival on non-Bt corn for the standard susceptible strain (mean percentage $\pm S E$, Lab-RR: $52.5 \pm 6.4$; IrmaF: $58.1 \pm 4.9$; Lab-SS: $61.9 \pm 2.4 ; F=0.48 ; \mathrm{df}=2,45 ; P=0.40)$. The Lab-RR strain was therefore considered to be appropriate for use in the fitness cost studies.

\subsection{Developmental time, pupal weight and growth rate}

Life-history traits related to growth and development are shown in Fig. 1, and their analyses of variance are summarized in supporting information Table S1. Interactions between S. frugiperda genotype and sex were significant $(P<0.05)$ for some life history traits but not for others, and no clear pattern existed for any of the traits measured in either strain. However, when comparing the susceptible strain with the resistant strain and their progenies, pupal weight significantly increased for Lab-RR and heterozygous males, and growth rate was significantly higher for Lab-RR and heterozygous males and for heterozygous Rơ So females. Importantly, no major and significant fitness disadvantage in relation to MT susceptible strain was observed in either heterozygotes $\left(F_{1}\right.$ progenies

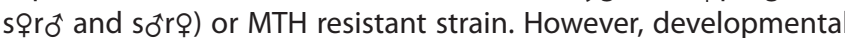

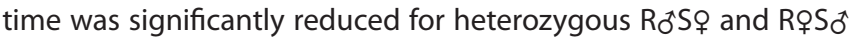
$(\mathrm{MTH} \times \mathrm{MT})$ males and for heterozygous Rơ So (Lab-RR $\times$ Lab-SS) males (Figs 1B and E).

\subsection{Fecundity and progeny production}

When assessing fecundity and progeny production, no significant differences were observed on the parameters daily egg mass weight, number of eggs per female and number of neonates per female $(F<1.45, P>0.23)$ within the matched pairs of resistant and susceptible strains (i.e. MTH versus MT and Lab-RR versus Lab-SS)

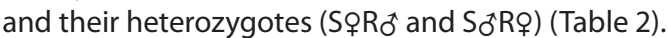

\subsection{Life table parameters}

No significant differences in the net reproductive rate $\left(R_{0}\right)$ and intrinsic rate of population increase $\left(r_{m}\right)$ were observed between the resistant and susceptible strains (Table 3 ). $R_{\circ}$ and $r_{\mathrm{m}}$ were higher for the heterozygotes derived from the Lab-RR $\times$ Lab-SS ${ }^{*}$ cross than for their homozygous parents (Table 3). Likewise, the mean generation time $(T)$ was not different in resistant and susceptible strains, but was significantly lower for MTHo $\times$ MT $\widehat{0}$.

\subsection{Larval respiration rate}

$\mathrm{CO}_{2}$ production differed between homozygous (resistant and susceptible) and heterozygous larvae $(F=20.07 ; \mathrm{df}=3,55$; $P<0.05$ ), with the $F_{1}$ hybrids (i.e. heterozygous larvae) showing a respiration rate higher than their resistant or susceptible homozygous parents (Fig. 2). Importantly, no significant differences in respiration rate were observed between the resistant and susceptible homozygotes (MTH versus MT and Lab-RR versus Lab-SS).

\subsection{Resistance stability}

Analysis of variance for larval survival rates obtained in the discriminating-concentration bioassays detected no significant differences among the seven generations tested for both MT-RS $(\mathrm{F}=0.66 ; \mathrm{df}=6,91 ; P=0.68)$ and Lab-RS $(F=0.77 ; \mathrm{df}=6,91$; $P=0.60)$ lines. The mean percentage survival remained near $20 \%$ in both strains (Fig. 3), indicating a constant proportion of homozygous resistant individuals in both strains, or that the resistance allele frequency remained stable during the generations tested.

\section{DISCUSSION}

Fitness and resistance stability in two $S$. frugiperda strains resistant to Cry1Fa with distinct genetic backgrounds were examined in this study. The results revealed no major differences in the net reproductive rate $\left(R_{0}\right)$ and the intrinsic rate of population increase $\left(r_{\mathrm{m}}\right)$ between the resistant and susceptible genotypes, a pattern that was consistent for fecundity measurements. Likewise, there were no differences between resistant and susceptible insects 

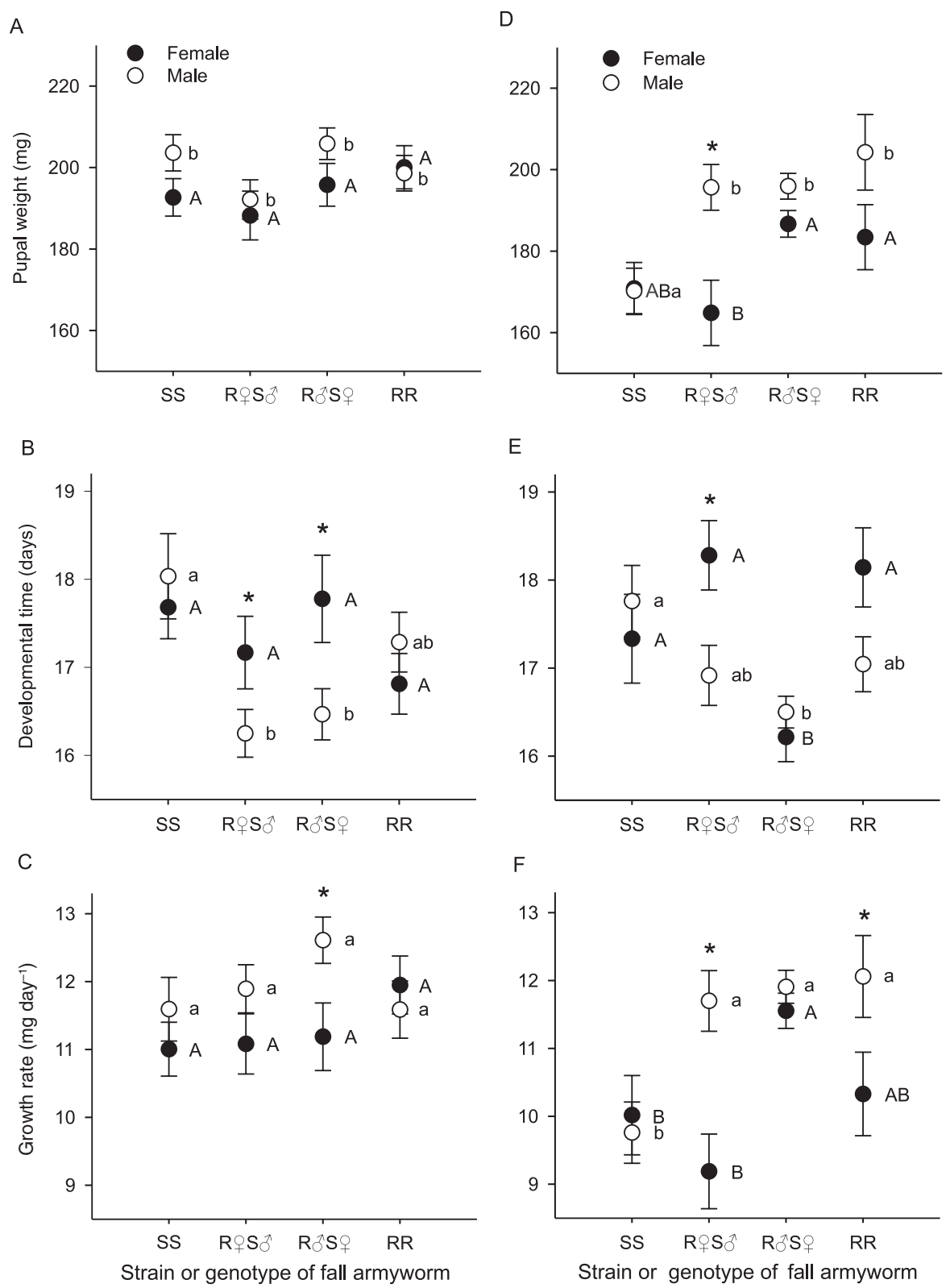

Figure 1. Life-history traits related to growth, development and body size (i.e. pupal weight) in two strains of Spodoptera frugiperda. Insect genotypes represent two Cry1Fa-resistant (MTH, Lab-RR) and Cry1Fa-susceptible (MT, Lab-SS) strains as well as $\mathrm{F}_{1}$ hybrid progenies from reciprocal crosses between them. Values are means \pm standard errors, $n=40-55$. (A, B, C) MTH (resistant) and MT (susceptible) strains and their reciprocal crosses. (D, E, F) Lab-RR (resistant) and Lab-SS (susceptible) strains and their reciprocal crosses. In each panel, black circles (females) with the same upper-case letter and white circles (males) with the same lower-case letter do not differ according to the LSD procedure protected by ANOVA (supporting information Table S1), $P>0.05$. Asterisks indicate significant difference $(P<0.05)$ between males and females of a given genotype.

in larval respiration rate experiments, and Cry1Fa resistance was stable over seven generations in the absence of selection in both resistant strains studied, indicating absence of fitness costs associated with resistance to Cry1Fa. These findings corroborate those reported for Cry1Fa-resistant S. frugiperda populations from Puerto Rico and Brazil. ${ }^{13-15}$

Life table statistics showed that the heterozygous individuals had similar or even higher demographic performance compared with susceptible homozygotes, which was also previously reported ${ }^{13}$ and indicates the potential presence of hybrid vigor. The similarity between resistant strains from Puerto Rico and Brazil $^{18,22,23,31}$ is indicative of a common mechanism that confers high levels of Cry1Fa resistance in S. frugiperda, which appears to be related to the reduced expression of alkaline phosphatase, a receptor protein from the insect midgut that is involved in the mode of action of Cry toxins, ${ }^{41}$ with minimal pleiotropic effects of the resistance allele on fitness of resistant individuals. ${ }^{13-15}$ These results have key implications for resistance management because a lack of fitness costs favors the permanence of resistance alleles in the absence of selection pressure, potentially maintaining the frequency of resistant individuals in the field. ${ }^{7,10,42}$ This could threaten pyramid crops producing Cry 1 protein because of cross-resistance between closely related $B t$ toxins. ${ }^{43}$ For example, a strain of fall armyworm that had field-relevant resistance to Cry1Fa corn rapidly evolved resistance to Cry1A.105 + Cry2Ab corn when exposed to this pyramid in the laboratory. ${ }^{44}$ For insects 


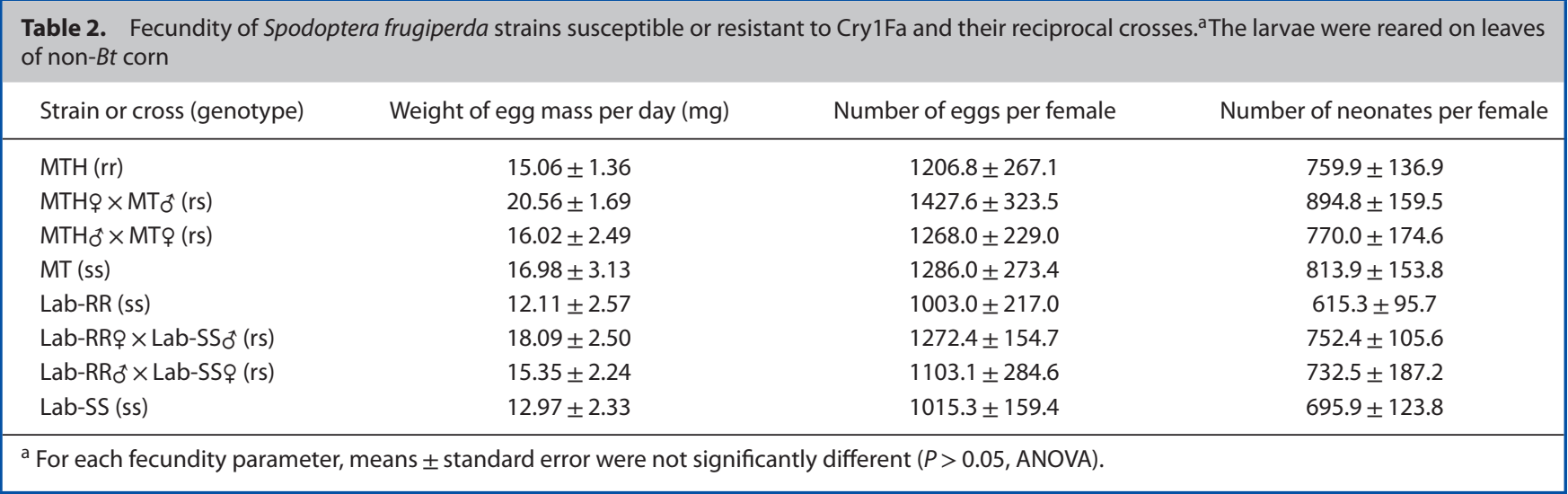

Table 3. Population growth rates (mean and $95 \%$ confidence interval) of Spodoptera frugiperda strains susceptible or resistant to Cry $1 \mathrm{Fa}$ and their reciprocal crosses. The larvae were reared on leaves of non-Bt corn

\begin{tabular}{|c|c|c|c|}
\hline \multirow[b]{2}{*}{ Strain or cross (genotype) } & \multicolumn{3}{|c|}{ Demographic parameter ${ }^{\mathrm{a}}$} \\
\hline & $R_{\mathrm{o}}$ & $r_{\mathrm{m}}$ & $T$ \\
\hline MTH (rr) & $178.6(104.4-252.9)$ a & $0.202(0.179-0.224) \mathrm{a}$ & $25.7(24.5-26.8) a b$ \\
\hline 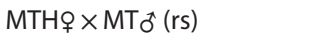 & $230.5(119.3-341.8)$ a & $0.218(0.192-0.244)$ a & $24.9(24.1-25.8) b$ \\
\hline $\mathrm{MTH}_{\mathrm{O}^{+}} \times \mathrm{MT} Q(\mathrm{rs})$ & $165.9(91.0-240.9)$ a & $0.194(0.172-0.216) \mathrm{a}$ & $26.3(25.2-27.4) \mathrm{a}$ \\
\hline MT (ss) & $183.0(101.9-264.2) \mathrm{a}$ & $0.197(0.174-0.220) \mathrm{a}$ & $26.4(25.1-27.7) a$ \\
\hline Lab-RR (rr) & $144.7(92.3-197.2) b$ & $0.183(0171-0.195) b$ & $27.1(26.6-27.6) a$ \\
\hline Lab-RRo $\times$ Lab-SSơ (rs) & $223.8(155.4-292.2) \mathrm{a}$ & $0.207(0.191-0.222) \mathrm{a}$ & $26.1(25.1-27.2) \mathrm{a}$ \\
\hline Lab-RRơ × Lab-SSo (rs) & $178.6(75.3-281.4) a b$ & $0.186(0.163-0.210) a b$ & $27.8(25.0-27.2) a$ \\
\hline Lab-SS (ss) & $148.3(90.3-206.4) b$ & $0.180(0.166-0.195) b$ & $27.7(27.1-28.2) a$ \\
\hline
\end{tabular}

resistant to one toxin in a two-toxin plant, the plant does not act as a pyramid. ${ }^{45}$

It is important to note that the optimal environmental conditions for development of $S$. frugiperda using corn leaves might have affected the non-expression of costs of resistance, which may be more apparent under unfavorable conditions for the individuals. ${ }^{5,27,46}$ Nevertheless, our results are consistent with previous studies on fitness costs of Cry1Fa resistance in $S$. frugiperda using artificial diet, corn, soybean and cotton as larval food sources, which also failed to identify costs associated with resistance. ${ }^{13-15}$ Future studies should focus on the reproductive behavior of S. frugiperda to document further whether Cry1Fa resistance really does not come at a cost for this species. Additionally, future efforts should try to identify ecological factors that may alter fitness costs to improve our ability to manage the frequency or the spread of resistance alleles and perhaps to find opportunities for resistance mitigation.

We observed no differences in the larval respiration rates between resistant and susceptible strains, indicating no detectable change in metabolic rate associated with resistance as would be expected when detoxification enzymes are involved. Thus, Cry1Fa resistance does not seem to affect reallocation of energy to other functions, as growth and reproduction rates were not reduced in the resistant insects, which is in agreement with a study using Cry1Ac-resistant Spodoptera exigua. ${ }^{16}$ It is possible that the lack of detectable changes in metabolic rates is related to the mechanism of Cry1Fa resistance in S. frugiperda, which seems to involve reduced toxin binding to and reduced expression of the alkaline phosphate receptor, ${ }^{41,47}$ having minimal negative effects on fitness of resistant individuals. ${ }^{13-15}$

Although insecticide resistance provides a selective advantage in the presence of the xenobiotic, resistance alleles are rarely fixed in natural populations and tend to decrease in the absence of the selective agent. ${ }^{2}$ However, when resistance alleles carry no fitness costs, they tend to remain in the population, thus stabilizing resistance. ${ }^{42,48}$ Our study provided evidence that Cry1Fa resistance remains stable for seven generations in the absence of selection, which is consistent with the lack of costs on the life history traits studied. Similar results were obtained in a field-derived S. frugiperda population from Puerto Rico, providing consistent evidence for the lack of strong fitness costs associated with the resistance allele. ${ }^{13,14}$

Several factors have contributed to the rapid evolution of resistance to Cry1Fa in S. frugiperda populations from Brazil, including the incomplete recessiveness, ${ }^{20,22,23}$ low rates of adoption of the recommended refuge and lack of fitness costs of resistance to Cry1Fa in S. frugiperda. ${ }^{13,15}$ In tropical areas such as Brazil, the risk of resistance development is further increased by high pest pressure, multiple generations per year, local agricultural practices (multiple crop cycles per year), the presence of the same technologies in different crops, consistent low adoption of recommended refuge (or its low effectiveness for resistance management owing to insecticide applications) and lack of grower integrated pest management (IPM) implementation. These factors help to explain 

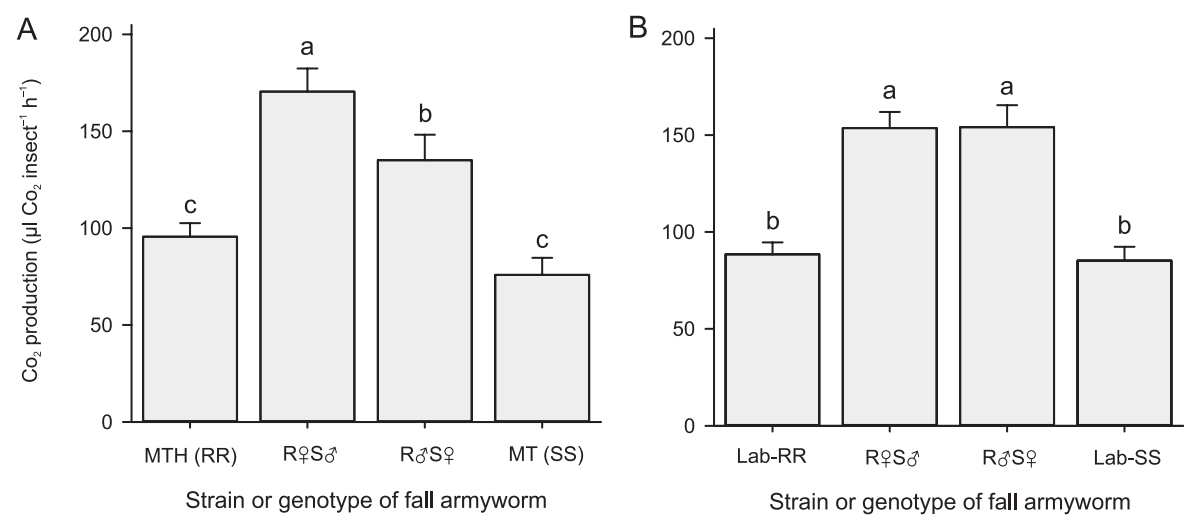

Figure 2. Production of $\mathrm{CO}_{2}\left(\mu \mathrm{L} \mathrm{CO}\right.$ insect $\left.^{-1} \mathrm{~h}^{-1}\right)$ by larvae of four Spodoptera frugiperda genotypes. (A) Cry1Fa-resistant (MTH) and Cry1Fa-susceptible (MT) homozygous strains and their reciprocal crosses; (B) Cry1Fa-resistant (Lab-RR) and Cry1Fa-susceptible (Lab-SS) homozygous strains and their reciprocal crosses. Means ( \pm standard error) followed by the same letters do not differ according to the LSD procedure protected by ANOVA, $P>0.05$, $n=14$.

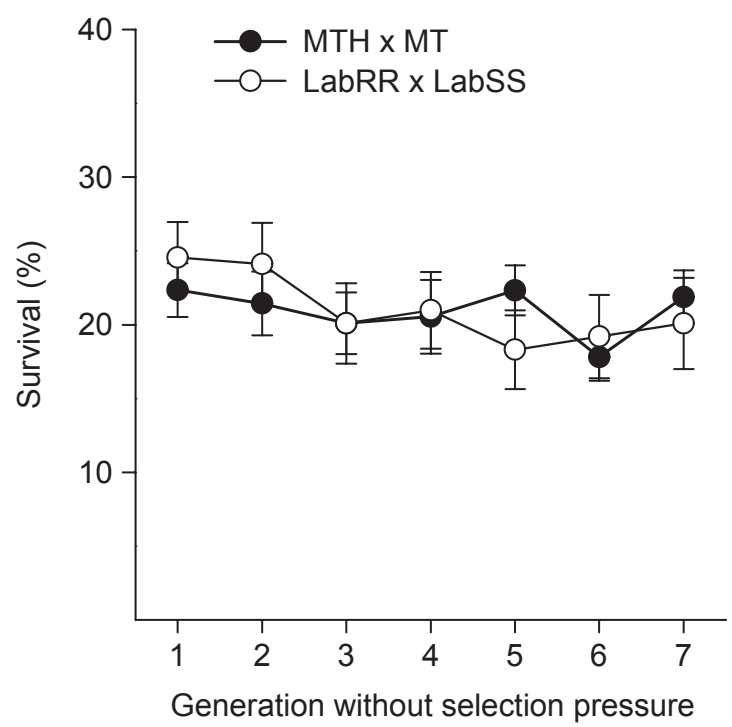

Figure 3. Stability of Cry1Fa resistance over seven generations in two lines (MT-RS and Lab-RS) of Spodoptera frugiperda. The lines resulted from two sets of reciprocal crosses between two Cry1Fa-selected and Cry1Fa-susceptible strains, MTH (RR) $\times$ MT (SS) and LabRR $\times$ LabSS. Values are means ( \pm standard error) of percentage survival of neonates exposed to a discriminating concentration of Cry1Fa for seven days using diet surface bioassays.

why resistance of S. frugiperda against Cry1Fa evolved relatively quickly in Brazil. Furthermore, the ability of this insect species to develop resistance against pest control tactics ${ }^{49}$ indicates that all biotechnologies are at risk, and that IPM strategies are necessary to help preserve the durability of the technologies that remain effective for pest control.

The most effective strategy to mitigate resistance is to implement several practices simultaneously to delay its occurrence, as recognized long ago..$^{50}$ For increased effectiveness, these practices should be deployed prior to resistance development. ${ }^{4}$ To this end, the implementation of insect resistance management (IRM) programs as a component of a broader IPM program is recommended. Basic principles of such programs should include pest complex monitoring in the field for changes in population density, focus on economic damage, resistance mitigation and integration of multiple strategies of control. ${ }^{51}$ Importantly, Bt technologies should be seen as a pest management tool and should be used within an intensive management program including practices of IPM and IRM. ${ }^{52,53}$ The paradigm that biotechnologies should be able to sustain themselves in tropical environments needs to be challenged, and programs focused on insect resistance management need to be implemented. To that extent, effective refuge areas (focused on producing susceptible insects, and not yet another yield plot) need to be implemented, along with multiple IPM approaches to reduce pest pressure levels and intervention with chemical applications to delay resistance development.

\section{CONCLUSION}

We found no fitness costs associated with Cry1Fa resistance in two S. frugiperda strains with distinct genetic backgrounds representative of Brazilian populations. This finding is consistent with the non-reversal trend in the proportion of resistant individuals in the laboratory, indicating that the resistance is likely to be stable in the absence of selective pressure under field settings and that the intensity of natural selection against the resistance alleles is likely weak, which helps to explain their quick rise in field populations of S. frugiperda. The unfavorable profile of fall armyworm resistance to $B t$ crops documented so far, together with the consistently low adoption of refuge (or its low effectiveness for resistance management owing to insecticide applications), as well as the lack of IPM implementation, places all biotechnologies at risk and needs to be improved if we are to sustain the utility of $B t$ proteins for pest management.

\section{ACKNOWLEDGEMENTS}

We thank the DuPont Young Professor Grant program, the National Council of Scientific and Technological Development (CNPq), the CAPES Foundation and PEC-PG System (Brazilian Ministry of Education) and the Minas Gerais State Foundation for Research Aid (FAPEMIG) for the financial support provided. Josemar Foresti and Paulo Roberto da Silva from Dupont Pioneer assisted in the field collections of insect populations and provision some of the rearing materials, which we greatly appreciated. The constructive criticisms provided by three anonymous reviewers helped to improve the manuscript, and their time and efforts are acknowledged here. 


\section{SUPPORTING INFORMATION}

Supporting information may be found in the online version of this article.

\section{REFERENCES}

1 Foster SP, Young S, Williamson MS, Duce I, Denholm I and Devine GJ, Analogous pleiotropic effects of insecticide resistance genotypes in peach-potato aphids and houseflies. Heredity 91:98-106 (2003).

2 Coustau C, Chevillon C and ffrench-Constant R, Resistance to xenobiotics and parasites: can we count the cost? Trends Ecol Evol 15:378-383 (2000).

3 Arnaud $L$ and Haubruge $E$, Insecticide resistance enhances male reproductive success in a beetle. Evolution 56:2435-2444 (2002).

4 Gould F, Sustainability of transgenic insecticidal cultivars: integrating pest genetics and ecology. Annu Rev Entomol 43:701 - 726 (1998).

5 Raymond B, Sayyed AH and Wright DJ, Genes and environment interact to determine the fitness costs of resistance to Bacillus thuringiensis. Proc R Soc B Biol Sci 272:1519-1524 (2005).

6 Gassmann AJ, Carriere $Y$ and Tabashnik BE, Fitness costs of insect resistance to Bacillus thuringiensis. Annu Rev Entomol 54:147-163 (2009).

7 Carrière $Y$ and Tabashnik BE, Reversing insect adaptation to transgenic insecticidal plants. Proc R Soc B Biol Sci 268:1475-1480 (2001).

8 Carrière $\mathrm{Y}$, Crowder DW and Tabashnik BE, Evolutionary ecology of insect adaptation to Bt crops. Evol Applic 3:561 - 573 (2010).

9 Tabashnik BE, Dennehy $\mathrm{TJ}$ and Carrière $\mathrm{Y}$, Delayed resistance to transgenic cotton in pink bollworm. Proc Natl Acad Sci USA 102:15 389-15393 (2005).

10 Gould F, Cohen MB, Bentur JS, Kennedy GG and Van Duyn J, Impact of small fitness costs on pest adaptation to crop varieties with multiple toxins: a heuristic model. J Econ Entomol 99:2091 - 2099 (2006).

11 Pereira EJG, Storer NP and Siegfried BD, Fitness costs of Cry1F resistance in laboratory-selected European corn borer (Lepidoptera: Crambidae). J Appl Entomol 135:17-24 (2011).

12 Crespo ALB, Spencer TA, Tan SY and Siegfried BD, Fitness costs of Cry $1 \mathrm{Ab}$ resistance in a field-derived strain of Ostrinia nubilalis (Lepidoptera: Crambidae). J Econ Entomol 103:1386-1393 (2010).

13 Vélez AM, Spencer TA, Alves AP, Crespo ALB and Siegfried BD, Fitness costs of Cry1F resistance in fall armyworm, Spodoptera frugiperda. $J$ Appl Entomol 138:315-325 (2014).

14 Jakka SR, Knight VR and Jurat-Fuentes JL, Fitness costs associated with field-evolved resistance to Bt maize in Spodoptera frugiperda (Lepidoptera: Noctuidae). J Econ Entomol 107:342-351 (2014).

15 Horikoshi RJ, Bernardi O, Bernardi D, Okuma DM, Farias JR, Miraldo LL et al., Near-isogenic Cry1F-resistant strain of Spodoptera frugiperda (Lepidoptera: Noctuidae) to investigate fitness cost associated with resistance in Brazil. J Econ Entomol 10.1093/jee/tov387 in press (2016).

16 Dingha BN, Moar WJ and Appel AG, Effects of Bacillus thuringiensis Cry $1 \mathrm{C}$ toxin on the metabolic rate of Cry1C resistant and susceptible Spodoptera exigua (Lepidoptera: Noctuidae). Physiol Entomol 29:409-418 (2004).

17 Li HR, Oppert B, Higgins RA, Huang FN, Zhu KY and Buschman LL, Comparative analysis of proteinase activities of Bacillus thuringiensis-resistant and -susceptible Ostrinia nubilalis (Lepidoptera: Crambidae). Insect Biochem Mol 34:753-762 (2004).

18 Storer NP, Babcock JM, Schlenz M, Meade T, Thompson GD, Bing JW et al., Discovery and characterization of field resistance to $B t$ maize: Spodoptera frugiperda (Lepidoptera: Noctuidae) in Puerto Rico. J Econ Entomol 103:1031-1038 (2010).

19 Huang F, Andow DA, Buschman LL, Qureshi JA, Meagher RL, Jr, Reisig $\mathrm{DD}$ et al., Cry1F resistance in fall armyworm Spodoptera frugiperda: single gene versus pyramided Bt maize. PLoS ONE 9:e112958 (2014).

20 Farias JR, Andow DA, Horikoshi RJ, Sorgatto RJ, Fresia P, dos Santos $\mathrm{AC}$ et al., Field-evolved resistance to Cry1F maize by Spodoptera frugiperda (Lepidoptera: Noctuidae) in Brazil. Crop Prot 64:150-158 (2014).

21 Dangal $V$ and Huang $F$, Fitness costs of Cry1F resistance in two populations of fall armyworm, Spodoptera frugiperda (JE Smith), collected from Puerto Rico and Florida. J Invertebr Pathol 127:81 - 86 (2015).

22 Leite NA, Mendes SM, Santos-Amaya OF, Santos CA, Teixeira TPM, Guedes RNC et al., Rapid selection and characterization of Cry1F resistance in a Brazilian strain of fall armyworm. Entomol Exp Applic 158:236-247 (2016).
23 Santos-Amaya OF, Tavares CS, Monteiro HM, Teixeira TPM, Guedes RNC, Alves AP et al., Genetic basis of Cry1F resistance in two Brazilian populations of fall armyworm, Spodoptera frugiperda. Crop Prot 81:154-162 (2016).

24 Kasten P, Jr, Precetti AACM and Parra JRP, Dados biológicos comparativos de Spodoptera frugiperda (J. E. Smith, 1797) em duas dietas artificiais e substrato natural. Rev Agric 53:68-78 (1978).

25 Cruz JC, Pereira Filho IA, Alvarenga RC, Gontijo Neto MM, Viana JHTM, Oliveira MF et al., Cultivo do Milho. Embrapa Milho e Sorgo, Sete Lagoas, MG, Brazil (2010).

26 Bergelson $\mathrm{J}$ and Purrington $\mathrm{CB}$, Surveying patterns in the cost of resistance in plants. Am Nat 148:536-558 (1996).

27 Raymond B, Wright DJ and Bonsall MB, Effects of host plant and genetic background on the fitness costs of resistance to Bacillus thuringiensis. Heredity 106:281 - 288 (2011).

28 Wang P, Zhao JZ, Rodrigo-Simon A, Kain W, Janmaat AF, Shelton AM et al., Mechanism of resistance to Bacillus thuringiensis toxin Cry1 AC in a greenhouse population of the cabbage looper, Trichoplusia ni. Appl Environ Microbiol 73:1199-1207 (2007).

29 Santos-Amaya OF, Characterization of resistance to Cry1Fa in Brazilian populations of Spodoptera frugiperda. PhD dissertation, Universidade Federal de Viçosa, Viçosa, Brazil (2014).

30 Marçon PCRG, Young LJ, Steffey KL and Siegfried BD, Baseline susceptibility of European corn borer (Lepidoptera: Crambidae) to Bacillus thuringiensis toxins. J Econ Entomol 92:279-285 (1999).

31 Vélez AM, Spencer TA, Alves AP, Moellenbeck D, Meagher RL, Chirakkal $\mathrm{H}$ et al., Inheritance of $\mathrm{Cry} 1 \mathrm{~F}$ resistance, cross-resistance and frequency of resistant alleles in Spodoptera frugiperda (Lepidoptera: Noctuidae). Bull Entomol Res 103:700-713 (2013).

32 Birch LC, The intrinsic rate of natural increase of an insect population. $J$ Anim Ecol 17:15-26 (1948).

33 Carey JR, Applied Demography for Biologists with Special Emphasis on Insects. Oxford University Press, Oxford, UK (1993).

34 Maia AHN, Luiz AJB and Campanhola C, Statistical inference on associated fertility life table parameters using jackknife technique: computational aspects. J Econ Entomol 93:511-518 (2000).

35 Base SAS 9.4 Procedures Guide: Statistical Procedures. SAS Institute, Cary, NC (2013).

36 Guedes RNC, Oliveira EE, Guedes NMP, Ribeiro B and Serrao JE, Cost and mitigation of insecticide resistance in the maize weevil, Sitophilus zeamais. Physiol Entomol 31:30-38 (2006).

37 Finney DJ, Probit Analysis. Cambridge University Press, Cambridge, UK (1971).

38 Robertson JL, Russel RM, Preisler HK and Savin NE, Bioassays with Arthropods, 2nd edition. CRC Press, Boca Raton, FL (2007).

39 Efron B, The Jackknife, the Bootstrap and Other Resampling Plans. Society for Industrial and Applied Mathematics, Philadelphia, PA (1982).

40 Meyers JS, Ingersoll CG, McDonald LL and Boyce MS, Estimating uncertainty in population growth rates: jackknife vs. bootstrap techniques. Ecology 67:1156-1166 (1986).

41 Jakka SRK, Gong L, Hasler J, Banerjee R, Sheets JJ, Narva K et al., Field-evolved Mode 1 resistance of the fall armyworm to transgenic Cry1Fa-expressing corn associated with reduced Cry1Fa toxin binding and midgut alkaline phosphatase expression. Appl Environ Microbiol 82:1023-1034 (2016).

42 Roush RT and Mckenzie JA, Ecological genetics of insecticide and acaricide resistance. Annu Rev Entomol 32:361 - 380 (1987).

43 Carrière $\mathrm{Y}$, Crickmore $\mathrm{N}$ and Tabashnik $\mathrm{BE}$, Optimizing pyramided transgenic Bt crops for sustainable pest management. Nat Biotechnol 33:161 - 168 (2015).

44 Santos-Amaya OF, Rodrigues JVC, Souza TC, Tavares CS, Campos SO, Guedes RNC et al., Resistance to dual-gene Bt maize in Spodoptera frugiperda: selection, inheritance, and cross-resistance to other transgenic events. Sci Rep 5:18243 (2015).

45 Carrière $Y$, Fabrick JA and Tabashnik BE, Can pyramids and seed mixtures delay resistance to Bt crops? Trends Biotechnol DOI: 10.1016/j.tibtech.2015.12.011 in press (2016).

46 Janmaat AF and Myers J, Rapid evolution and the cost of resistance to Bacillus thuringiensis in greenhouse populations of cabbage loopers, Trichoplusia ni. Proc R Soc B Biol Sci 270:2263-2270 (2003).

47 Jurat-Fuentes JL, Karumbaiah L, Jakka SRK, Ning CM, Liu CX, Wu KM et al., Reduced levels of membrane-bound alkaline phosphatase are common to lepidopteran strains resistant to Cry toxins from Bacillus thuringiensis. PLoS ONE 6: e17606 (2011).

48 Tabashnik BE, Evolution of resistance to Bacillus thuringiensis. Annu Rev Entomol 39:47-79 (1994). 
49 Whalon ME, Mota-Sanchez D and Hollingworth RM, Arthropod Pesticide Resistance Database. [Online]. Available: http://www.pesticid eresistance.org/index.php [15 April 2016].

50 Mcgaughey WH and Whalon ME, Managing insect resistance to Bacillus thuringiensis toxins. Science 258:1451-1455 (1992).

51 Tabashnik BE and Croft BA, Managing pesticide resistance in crop-arthropod complexes: interactions between biological and operational factors. Environ Entomol 11:1137-1144 (1982).

52 Onstad DW, Major issues in insect resistance management, in Insect Resistance Management: Biology, Economics, and
Prediction, ed. by Onstad DW. Academic Press, San Diego, CA, pp. 1-23 (2014)

53 Tabashnik BE and Carrière $Y$, Successes and failures of transgenic $B t$ crops: global patterns of field-evolved resistance, in Bt Resistance: Characterization and Strategies for GM Crops Producing Bacillus thuringiensis Toxins, ed. by Soberón M, Gao A and Bravo A. CABI, Wallingford, Oxon, UK, pp. 1-14 (2015). 